\title{
Involucramiento de las organizaciones en la construcción de paz
}

Envolvimento das empresas na construção da paz

Involvement of organizations in peacebuilding

\section{Sandra I. Fuentes Martinez}

- Maestra en Derechos Humanos y Cultura de Paz por Pontificia Universidad Javeriana, Cali, Colombia.

- Posgraduada en Comunicación para la Dinámica Organizacional.

- Especialista en Estudios de Paz y Conflicto con énfasis en Medio Ambiente, Género, Negociación y Acuerdos por la University for Peace de la Organización de las Naciones Unidas (ONU) en San José, Costa Rica.

- Catedrática en universidades de Iberoamérica y docente de la Facultad de Comunicación de la Pontificia Universidad Javeriana, Bogotá, Colombia.

- Dirigió durante 15 años el posgrado en Comunicación Organizacional de la Pontificia Universidad Javeriana de Bogotá.

- Directora general del Grupo SAF-Colombia, firma de consultoría en comunicación, cultura y sostenibilidad corporativa.

- Autora y coautora de varios libros en gestión y comunicación.

- Columnista en la sección de análisis del diario La República.

- E-mail: sfuentes@gruposaf.com 


\section{Resumen}

La realidad del contexto en Latinoamérica y en el Caribe demanda el involucramiento activo de las organizaciones en la construcción de la paz, proceso que es complejo, a largo plazo y requiere de la participación de todos los actores de la sociedad; de manera particular las empresas tienen la capacidad, el deber, el poder y los recursos para impulsar esta transformación. Este involucramiento implica la comprensión y adopción de lineamientos globales que orientan el trabajo por la paz con efectos en el respeto de los Derechos Humanos y en el Desarrollo Sostenible. Así mismo, necesita de la gestión estratégica de la comunicación para recomponer el tejido social al afianzar la interrelación de la organización con la sociedad, al construir vínculos basados en la confianza, y al desarmar la palabra para construir una nueva narrativa de paz que permita consolidar una sociedad armoniosa y pacífica.

\section{PALABRAS CLAVE: COMUNICACIÓN・CONSTRUCCIÓN DE PAZ・DERECHOS HUMANOS・DESARROLLO SOSTENIBLE.}

\section{Abstract}

The reality of Latin America and the Caribbean requires active involvement of organizations in peacebuilding, a complex, long term process that requires the participation of all society actors. In particular, companies that have the ability, the duty, the power and the resources to promote this transformation. This involvement implies understanding and adopting international guidelines to orient work for peace, reflecting into the respect towards human rights and sustainable development. Similarly, it requires strategic communication management to rebuild social fabric, strengthening the interrelation between organization and society to build ties based on trust and disarm words in order to construct a new narrative of peace that leads to a harmonious and peaceful society.

\section{KEYWORDS: COMMUNICATION • PEACEBUILDING • HUMAN RIGHTS・SUSTAINABLE DEVELOPMENT.}

\section{Resumo}

A realidade da América Latina e do Caribe exige o envolvimento ativo das organizações na construção da paz, processo que é complexo, de longo prazo e requer a participação de todos os atores da sociedade. De maneira particular, as empresas que têm a capacidade, o dever, o poder e os recursos para promover essa transformação. Esse envolvimento implica a compreensão e a adoção de diretrizes globais que orientem o trabalho pela paz, com reflexos no respeito aos direitos humanos e ao desenvolvimento sustentável. Da mesma forma, requer gestão estratégica da comunicação para reconstruir o tecido social, fortalecendo a interrelação da organização com a sociedade ao construir vínculos baseados na confiança e ao desarmar a palavra para construir uma nova narrativa de paz que consolide uma sociedade harmoniosa e pacífica. 
ANO 15 • NÚMERO 28 • 1ํㅗㅌ. 2018 • ORGANICOM

\section{INTRODUCCIÓN}

“El trabajo por la paz es una tarea conjunta, universal, y sus logros serán el resultado de una gran convergencia mundial, de mucha gente actuando sobre diferentes y múltiples frentes, pero cada vez con mayor interconexión entre ellas y con mayor comprensión del significado de lo que hacen los demás." (Fisas, 2002, p. 35)

$\mathrm{E}$ I trabajo conjunto por la paz requiere del involucramiento de todos los actores de la sociedad y de manera particular las organizaciones, más aún cuando se está inmerso en una sociedad de organizaciones. El contexto actual tiene demandas sociales, ambientales y económicas que necesitan respuesta urgente de la sociedad y, como son actores claves, se requiere que las empresas sean protagonistas responsables, comprometidas e involucradas en transformar realidades, teniendo como base el respeto de los Derechos Humanos y la construcción de la paz. Este artículo busca evidenciar la importancia del involucramiento de las organizaciones en la construcción de la paz de un país o de una región, así mismo realizar una reseña de los lineamientos globales que les brindan a las empresas insumos para que con su gestión contribuyan a la cultura de la paz. Cabe destacar que esta reflexión se realiza en el marco del proceso de paz de Colombia, por lo cual se hace una breve descripción de este y de lo que se espera de las empresas en la implementación de los Acuerdos. Finalmente, se presenta el rol que debe desempeñar la comunicación en el proceso de construcción de paz para recomponer el tejido social y construir confianza.

\section{COLOMBIA Y AMÉRICA LATINA: UN TERRITORIO PROPICIO PARA CONSTRUIR LA PAZ}

Durante cinco décadas, Colombia tuvo un conflicto armado interno que se caracterizó por ser: prolongado, ${ }^{1}$ complejo, ${ }^{2}$ con enormes diferencias regionales, ${ }^{3}$ con raíces políticas ${ }^{4}$ y atroz. ${ }^{5}$ En el año 2012, se inició el proceso de paz, antecedido por tres intentos fallidos en los años 1984, 1991 y 1999. El proceso que se realizó con las Fuerzas Armadas Revolucionarias de Colombia(FARC) se culminó con la firma de los acuerdos en el 2017. En él se escuchó a todas las partes involucradas en el conflicto, en el centro de este proceso y de los acuerdos estuvieron las víctimas, que en este caso ascendieron a 8.650.169 personas, según el registro de la Unidad de Víctimas del Gobierno Nacional. El reto que enfrenta un país después de un conflicto prolongado para avanzar hacia la construcción de la paz es recomponer el tejido social, y para ello debe recuperar la confianza junto a la esperanza de un futuro más prometedor. Este es uno de los aspectos que insistentemente ha resaltado el Padre Francisco de Roux (2016), dando el ejemplo de la capacidad de volver a confiar que tienen las víctimas y la esperanza que guardan en una Colombia que se puede transformar. En varios escenarios, ha compartido apartes de relatos que hicieron las mujeres víctimas del conflicto en la mesa de negociación de La Habana: "Nosotras no vinimos aquí a invitar a que esta oscuridad siga, a que se acrecienten los odios, venimos a que trabajemos juntos, juntos por construir la luz y la paz en este país" (De Roux, 2016). "Los colombianos

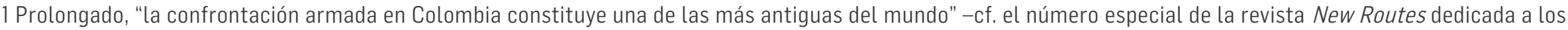
conflictos prolongados en el mundo y, en particular, el artículo de Marcus Nilsson e Joakim Kreutz (2010), "Protracted conflicts: issues or dynamics at stake?".

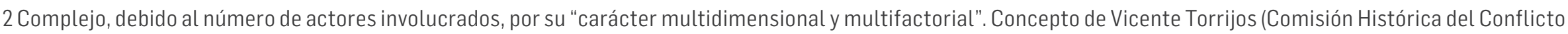
y sus Víctimas, 2015, p. 1)

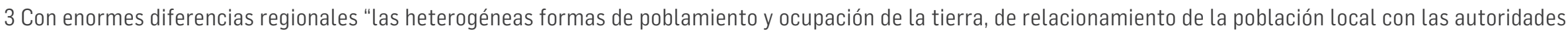

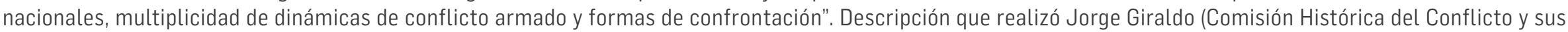
Víctimas, 2015, p. 45-47), integrante de la Comisión histórica del Conflicto y sus víctimas (CHCV).

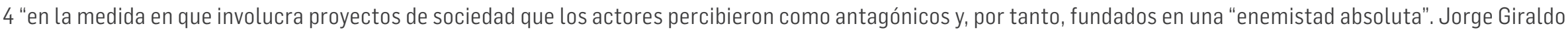
(Comisión Histórica del Conflicto y sus Víctimas, 2015, p. 45-47).

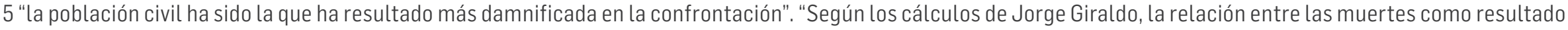

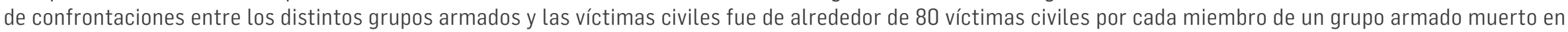

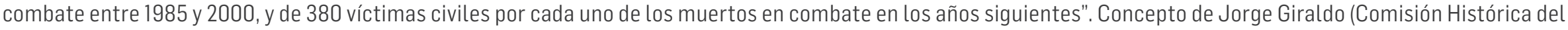
Conflicto y sus Víctimas, 2015, p. 30).
} 
somos capaces de emerger de ese barrial, nosotros somos capaces de volver a reconstruir este país, de volver a creer los unos en los otros, somos capaces de reconciliarnos, somos capaces de reconstruir este país, incluso de perdonarnos" (De Roux, 2016).

Tenemos muchos retos por delante, según el Padre de Roux; dos de ellos: recobrar la capacidad de vivir como seres humanos sin destruirnos los unos a los otros, sin rompernos y sin humillarnos; así mismo, ayudar al empresariado a comprender sus enormes responsabilidades en estos problemas de fondo, de equidad, de dignidad de la persona, pero también de compromiso con la paz del país.

Todos los colombianos han sido afectados por este conflicto en mayor o menor magnitud, y uno de los aspectos relevantes de cara a una construcción de un país en paz es la pérdida de confianza. Según Francisco Gutiérrez de la Comisión Histórica del Conflicto y sus Víctimas (2015), "el conflicto destruyó de manera masiva el tejido social, las tradiciones positivas y las redes de confianza [...] [y] tuvo un efecto deletéreo sobre la confianza de los colombianos en sus conciudadanos y en las instituciones" (p. 36). Este escenario de desconfianza no se da únicamente en Colombia por el conflicto armado interno, es una característica de América Latina, según el estudio de la Corporación Latinobarómetro (2017, p. 20):

El resultado de 2017 alcanza un promedio nacional más bajo desde que se empezara a medir en 1996, de sólo un 14\%. La confianza interpersonal ha llegado al punto más bajo en los últimos 20 años en la región de América Latina.

Son múltiples las problemáticas que tienen América Latina y el Caribe, que a su vez son formas de violencias y conflictos también prolongados. Según Fisas (2002, p. 44), los desafíos que tenemos adelante no son precisamente de carácter militar únicamente, ya que nos enfrentamos a la pobreza, degradación del medio ambiente, la corrupción, la falta de gobernabilidad, la violencia urbana, la exclusión política y social, la ausencia de los Derechos Humanos y otros aspectos que son la base de los conflictos contemporáneos. Se puede afirmar que el reto de recuperar la confianza no es exclusivo de Colombia, sino de América Latina, y que esta es la base para construir una convivencia pacífica porque las implicaciones de la desconfianza complejizan las relaciones, los intercambios y las vinculaciones de todo tipo, por la sospecha, el temor y el miedo. La confianza, por lo tanto, produce previsibilidad y certidumbre, y es a partir de ellas que es posible desarrollar un futuro distinto (Rojas, 2011, p. 12).

A esta visión se suma la de Lederach (2016), quien explica que, en procesos de conflicto, construir la paz será un proceso complejo en donde el pesimismo acompaña la desconfianza, porque "la gente desconfía de las palabras, y reivindica que quiere ver hechos" (Lederach, 2016, p. 130). Por tanto, el camino por recorrer hacia la construcción de una paz estable y duradera se va a enfrentar a "tres sentimientos predominantes: la sospecha, la indiferencia y la distancia [...] porque existe una profunda brecha [...] en cómo la paz y los procesos de cambio que siguen a los acuerdos operan y moldean el futuro" (Lederach, 2016, p. 117).

\section{LAS ORGANIZACIONES: PROTAGONISTAS EN LA CONSTRUCCIÓN DE PAZ}

La paz es igual a desarrollo, afirma Galtung (1985), y las empresas buscan y son una de las bases del desarrollo de los países; sin embargo, este se debe enmarcar en promover la dignidad humana. La construcción de la paz es un proceso a largo plazo, complejo, "mediante el cual una sociedad realiza una transición entre un pasado dividido y un futuro compartido [...] un proceso que redefine relaciones" (Bloomfield; Barnes; Huyse, 2003, p. 12).

Uno de los actores de la sociedad que aporta a la construcción de la paz para hacer posible un futuro compartido es la empresa, podemos soportar esta afirmación en los siguientes argumentos: 


\section{Por su impacto de la transformación social}

Este impacto se puede ver desde tres ópticas. La primera, de cobertura, porque un gran porcentaje de personas tiene relación con una o varias empresas al ser empleados, proveedores, usuarios o parte de su mercado. Esto posibilitaría tener un factor multiplicador para movilizar a una gran masa crítica de personas hacia la construcción de la paz. La segunda es la incidencia global en todos los ámbitos del país: económico, social, cultural, ambiental y tecnológico. Por esta razón, si se gestiona una cultura de paz desde las empresas, los efectos hacia la sociedad se revertirían de forma integral en todos estos ámbitos.

La tercera, porque estamos en una sociedad de organizaciones donde cada una debe asumir la responsabilidad total de su impacto sobre los empleados, el medio, los clientes y todas las personas y cosas que toca, según el concepto de Peter Drucker. Además, porque estas tienen la capacidad de formar sociedad, siendo un principio deontológico que es la responsabilidad social, porque más allá de crear bienes, las empresas tienen la capacidad y el deber de aportar a la creación de sociedades armónicas (Savater, 2014, p. 84).

\section{Por ser aliado y actor clave}

En la actualidad, las empresas son aliados clave para movilizar la sostenibilidad del planeta; por ejemplo, en varias ocasiones, el anterior Secretario General de la ONU, Ban Ki-Moon, hizo referencia a que "las empresas en el mundo tienen responsabilidad social y sustentabilidad, lo cual debe ser un modelo que se replique y aumente en su dimensión, para darle viabilidad al mundo actual y futuro" (CONEXIÓN COP, 2015). Existen a nivel global lineamientos para que las empresas respeten los DDHH y se sumen a la construcción de la paz. Por ejemplo, el gobierno colombiano considera que el sector empresarial es un actor clave para la sostenibilidad de la paz, y establece la estrategia de empresas y paz, planteando tres líneas de acción base: ${ }^{6}$ a) contar con lineamientos claros en cuanto a debida diligencia y acción sin daño; b) desarrollar alianzas público-privadas que contribuyan al desarrollo local y a la ampliación de la democracia; y c) emprender acciones encaminadas a la reconciliación.

\section{Por su poder en la construcción de paz}

Es un hecho que las empresas ejercen poder en la sociedad, y en un proceso de paz aún más, porque tienen la expectativa que con ello: se aumente la inversión extranjera en el país, se acelere el crecimiento económico, se incremente las ventas de su empresa, se reduzca el nivel de la pobreza en el país, y se mejore la distribución del ingreso. ${ }^{7}$ Adicionalmente, la experiencia en otros procesos de paz del mundo ha dejado de enseñanza que los empresarios tienen un rol importante tanto en las negociaciones como en la implementación de los acuerdos, esto lo sustenta Angelika Rettberg (2012), quien considera que:

Los empresarios tienen una capacidad de veto que define el alcance y la estabilidad de las políticas acordadas. En países en los que los empresarios han estado opuestos a los acuerdos, estos han tenido dificultades en términos de implementación. Cuando los empresarios deciden actuar en pro de la construcción de la paz, sus acciones pesan más que la de muchos otros actores sociales.

\section{Por la oportunidad en la coyuntura}

América Latina y el Caribe tienen la oportunidad de avanzar hacia una transformación integral que genere cambios sostenibles. Es por ello que no se puede dejar pasar esta coyuntura para generar conciencia en las empresas sobre la evolución que deben 
hacer con la finalidad de aportar integralmente a la construcción de una paz estable y duradera. La evolución anterior debe llevar a las empresas a realizar procesos de autoevaluación de la forma como acogen y respetan la ley, de como gestionan sus procesos para ser empresas socialmente responsables, de como llevan a la práctica el respeto de los Derechos Humanos, de como consolidan su cultura organizacional basada en principios y valores, así como su relacionamiento con sus diferentes grupos de interés para construir confianza. Estos son algunos de los aspectos, que, en esta coyuntura, se deben promover hacia una nueva evolución en el sector empresarial. Es una oportunidad que el sector empresarial tiene en sus manos para focalizar sus acciones y realizar un verdadero aporte a la construcción de la paz desde su gestión de sostenibilidad. El objetivo es lograr que las empresas evidencien que este aporte es parte de su gestión, y que se le integre de la misma manera como se les integran a los temas del mercado o de las finanzas.

\section{LINEAMIENTOS GLOBALES PARA APORTAR A LA CONSTRUCCIÓN DE LA PAZ Y DEL RESPETO A LOS DERECHOS HUMANOS}

La construcción de la paz es un proceso complejo - y más aún cuando los conflictos son prolongados -, donde la cultura de violencia se arraigó en la sociedad, y se requiere de una gran transformación que llevará generaciones. Por tanto, se debe tener en cuenta la visión de lineamientos ya existentes que pueden orientar este proceso para que sean comprendidos, asimilados y apropiados, y así avanzar en este cambio social.

En palabras de Lederach (2016, p. 149): "El cambio social constructivo y la construcción de la paz por sí misma promueven y deben utilizar múltiples procesos de cambio, que son transversales a los niveles y a las poblaciones afectadas por el conflicto". En este sentido, es necesario identificar políticas, lineamientos y parámetros existentes, que, desde el ámbito global, se les han propuesto a las empresas para que propicien y promuevan este cambio social constructivo, permitiendo aportar a la construcción de la paz y al respeto de los Derechos Humanos.

Los lineamientos globales analizados, de acuerdo con la Figura 1, provienen de la Organización de las Naciones Unidas (ONU), por su carácter internacional y su enfoque hacia la preservación y promoción de la paz, Derechos Humanos y el Desarrollo Sostenible.

Figura 1: Visión y lineamientos globales

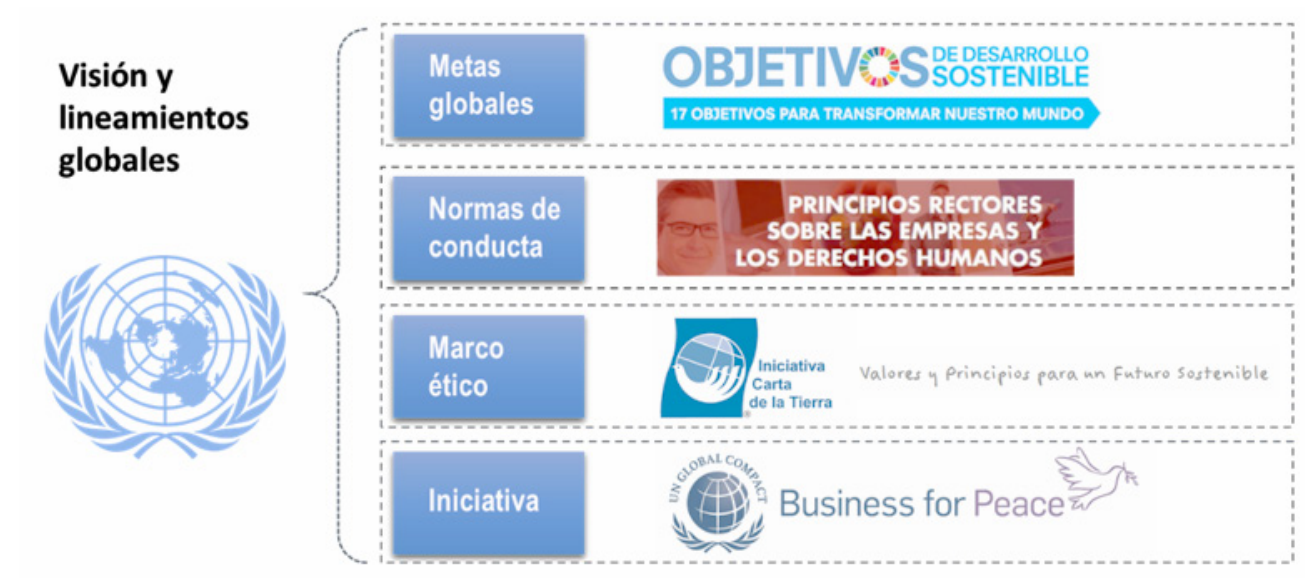

Fuente: Elaborado por la autora.

\section{METAS GLOBALES: OBJETIVOS DE DESARROLLO SOSTENIBLE}

Los Objetivos de Desarrollo Sostenible (ODS) son una visión de futuro resultado de una consulta pública entre países y de la evolución de los Objetivos de Desarrollo del Milenio. Los ODS son la nueva agenda mundial consensuada para el año 2030 , 


\title{
ANO 15 • NÚMERO 28 • 1ํㅗㅌ. 2018 • ORGANICOM
}

\section{INVOLUCRAMIENTO DE LAS ORGANIZACIONES EN LA CONSTRUCCIÓN DE PAZ}

que busca tener un gran alcance en la transformación del mundo, como se afirma en el documento de la cumbre de la Organización de las Naciones Unidas (2015, p. 4):

\begin{abstract}
Un mundo en el que sea universal el respeto de los Derechos Humanos y la dignidad de las personas, el estado de derecho, la justicia, la igualdad y la no discriminación; donde se respeten las razas, el origen étnico y la diversidad cultural, y en el que exista igualdad de oportunidades para que pueda realizarse plenamente el potencial humano y para contribuir a una prosperidad compartida; un mundo que invierta en su infancia y donde todos los niños crezcan libres de la violencia y la explotación; un mundo en el que todas las mujeres y niñas gocen de la plena igualdad entre los géneros y donde se hayan eliminado todos los obstáculos jurídicos, sociales y económicos que impiden su empoderamiento; un mundo justo, equitativo, tolerante, abierto y socialmente inclusivo en el que se atiendan las necesidades de los más vulnerables.
\end{abstract}

Para alcanzar esta meta, se proponen 17 objetivos de desarrollo sostenible con 169 metas conexas de carácter integrado e indivisible, conforme la Figura 2. Estos objetivos y metas conjugan las dimensiones del desarrollo sostenible: económica, social y ambiental; así como contempla cinco (5) esferas de importancia crítica para la humanidad y el planeta, estas son: las personas para desarrollar su potencial con dignidad e igualdad, el planeta para protegerlo con una gestión sostenible, la prosperidad para tener una vida plena y un progreso integral, la paz para propiciar sociedades justas e inclusivas y alianzas para movilizar e implementar la agenda 2030 (Organización de las Naciones Unidas, 2015).

Los 17 Objetivos de Desarrollo Sostenible son:

Figura 2: 17 objetivos de desarrollo sostenible

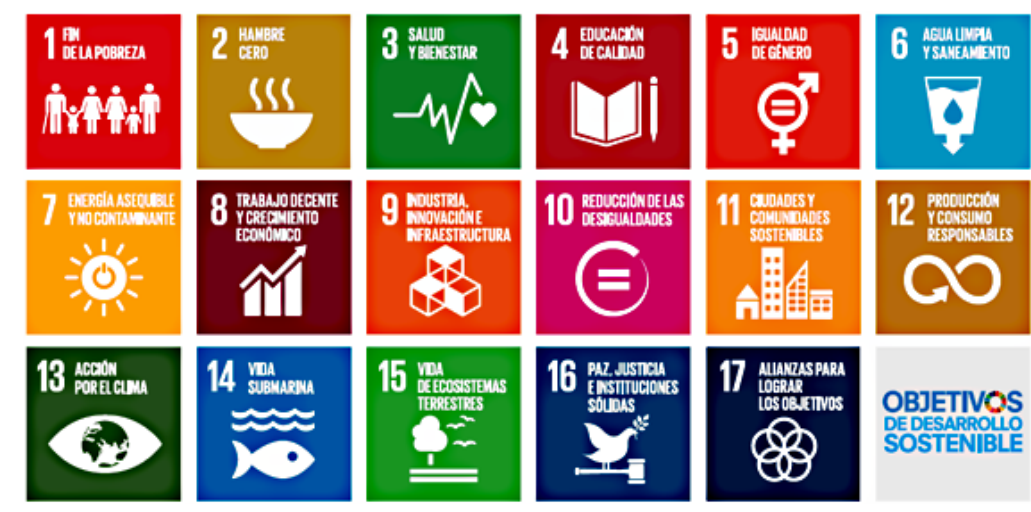

Fuente: Organización de las Naciones Unidas (s.d.).

Si bien todos los ODS aportan a la construcción de paz, de manera particular el Objetivo de Desarrollo Sostenible número 16 busca promover sociedades justas, pacíficas e inclusivas y una de sus metas es: reducir significativamente todas las formas de violencia y las correspondientes tasas de mortalidad en todo el mundo.

La implementación de los ODS inició en enero de 2016, se realiza de acuerdo con la realidad de cada país, y con el compromiso del gobierno, del sector privado, de la sociedad civil, del Sistema de Naciones Unidas y de las alianzas que se movilicen con "espíritu de solidaridad mundial". De manera especial, la Organización de las Naciones Unidas hace un llamado directo y explícito a las empresas de esta manera:

La actividad empresarial, la inversión y la innovación privadas son los grandes motores de la productividad, del crecimiento económico inclusivo y de la creación de empleo. Reconocemos la diversidad del sector privado, que incluye tanto a las microempresas como a las cooperativas y las multinacionales. Exhortamos a todas las empresas a que aprovechen su creatividad e innovación para resolver los problemas relacionados con el desarrollo sostenible. Fomentaremos un sector empresarial dinámico y eficiente, protegiendo al mismo tiempo los derechos laborales y los requisitos sanitarios y ambientales de conformidad con las normas y los acuerdos 
internacionales pertinentes y otras iniciativas que se estén llevando a cabo en esta esfera, como los Principios Rectores sobre las Empresas y los Derechos Humanos y las normas laborales de la Organización Internacional del Trabajo, la Convención sobre los Derechos del Niño, y los principales acuerdos ambientales multilaterales, para quienes sean parte en ellos. (Organización de las Naciones Unidas, 2015, p. 4)

El rol de las empresas en el cumplimiento de los ODS es fundamental, por ello, la tendencia creciente es que los gobiernos y gremios empresariales e industriales promuevan su aplicación; así mismo, se han generado guías de implementación, como la "SDG Compass" (Global Report Initiative; Pacto Global de las Naciones Unidas; World Business Council for sustainable Development, 2015), que orientan a las empresas como llevar a la acción los ODS e incorporarlos a su planeación estratégica, a través del entendimiento, definición de prioridades y alcance, integración de estos a los procesos del negocio, y el reporte de sus aportes en el informe de sostenibilidad. La apropiación e implementación de los ODS aún se realiza de manera incipiente, considerando el alcance y la generación de cambio de conciencia que se espera en las empresas y su aporte significativo al desarrollo sostenible.

\section{NORMAS DE CONDUCTA: PRINCIPIOS RECTORES DE DERECHOS HUMANOS}

Desde 2011, el Consejo de Derechos Humanos de las Naciones Unidas consagró a los Principios Rectores de Derechos Humanos como la norma de conducta a nivel mundial que se espera de todas las empresas en relación con los Derechos Humanos. Aunque no tiene carácter jurídico de obligatoriedad, resalta las repercusiones que tiene para la legislación nacional e internacional (Organización de las Naciones Unidas, 2011).

Los Principios Rectores (PR) buscan que el Estado tenga un compromiso político para proteger los Derechos Humanos y promover en las empresas la diligencia debida ${ }^{8}$, aclarando las responsabilidades que tienen de respetar y remediar o reparar si sus actividades tienen un impacto negativo sobre los Derechos Humanos.

Con los Principios Rectores, la responsabilidad de respetar los Derechos Humanos no es exclusividad de los actores estatales, sino que ahora los Estados miembros de Naciones Unidas ratifican que las empresas también tienen responsabilidad independiente.

Los 31 Principios Rectores están estructurados en tres pilares "proteger, respetar y remediar", que a su vez se clasifican en fundacionales porque enuncian y argumentan el deber, y operacionales ya que señalan las medidas a adoptar por los actores. El primer pilar PROTEGER dice: "Los Estados tienen la obligación de proteger los DDHH de posibles impactos de terceros, incluidas las empresas [...] a través de prevenir, investigar, castigar y reparar mediante medidas políticas, legislativas, de regulación y de sometimiento a la justicia" (Carneiro Freire et al., 2013, p. 20). En el caso de zonas afectadas por el conflicto se hace explícito en el PR $7{ }^{9}$ que el Estado debe proteger estas zonas de afectaciones de las actividades empresariales, porque "es frecuente que los peores abusos de los DDHH relacionados con empresas tengan lugar en estas zonas" (Carneiro Freire et al., 2013, p. 23).

El segundo pilar se concentra en las empresas para RESPETAR: "La obligación de respetar los DDHH, es una norma de conducta mundial aplicable para todas las empresas y es exigible como tal. No es opcional [...] La responsabilidad no solo abarca sus actividades, sino también las de terceros con los que tenga relaciones comerciales" (Carneiro Freire et al., 2013, p. 26).

8 Diligencia debida: "En el contexto de los Principios Rectores, la diligencia debida en materia de derechos humanos constituye un proceso continuo de gestión que una empresa prudente y razonable debe llevar a cabo a la luz de sus circunstancias (como el sector en el que opera, el contexto en que realiza su actividad, su tamaño, y otros factores) para hacer frente a su responsabilidad de respetar los derechos humanos" (Organización de las Naciones Unidas, 2012). 


\section{ANO 15 • NÚMERO 28 • 1ํㅗㅌ. 2018 • ORGANICOM}

\section{INVOLUCRAMIENTO DE LAS ORGANIZACIONES EN LA CONSTRUCCIÓN DE PAZ}

El respeto de los Derechos Humanos en las empresas implica un alto compromiso político que se ejerce en la práctica de la gobernanza y al generar una cultura organizacional en función de este objetivo. Este compromiso contempla de acuerdo con la Figura 3.

Figura 3: Compromiso político de las empresas -Principios Rectores de DDHH

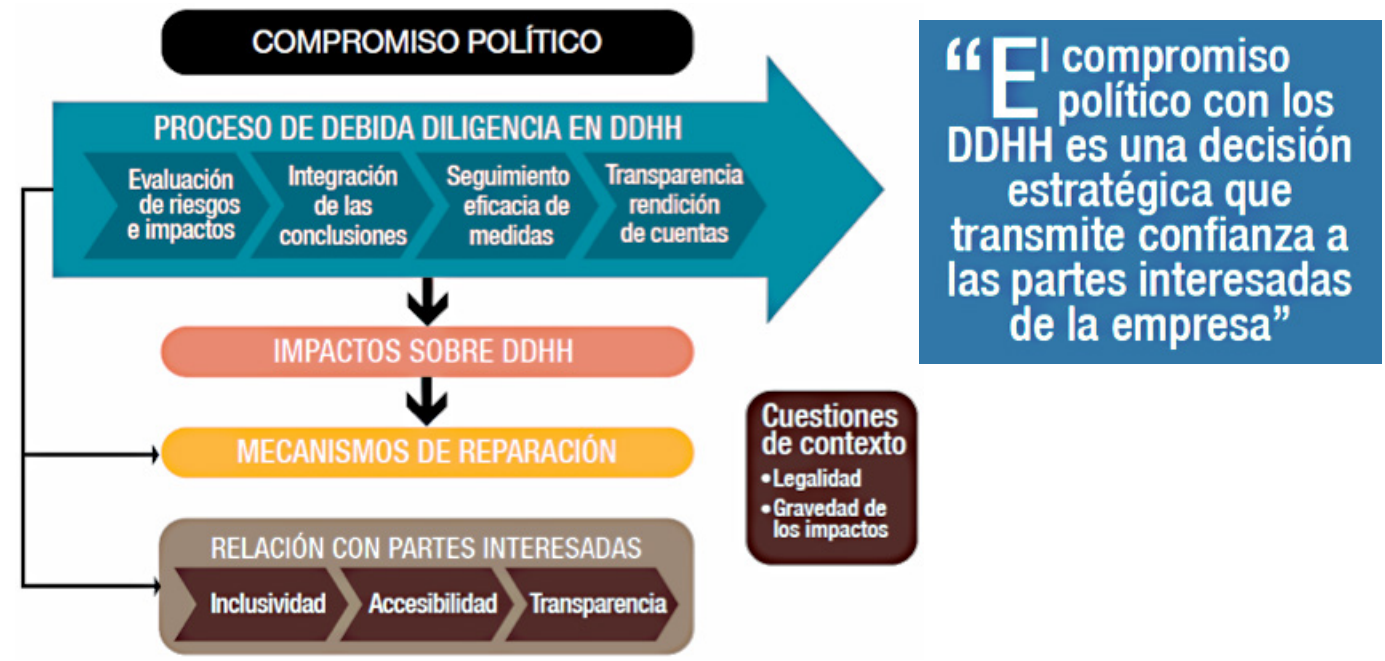

Fuente: Carneiro Freire et al. (2013, p. 29).

El tercer pilar es REMEDIAR, si bien el objetivo de los Principios Rectores es prevenir e identificar posibles riesgos de posibles afectaciones a los Derechos Humanos, la ONU propuso que deben existir dos deberes: "el deber de proteger a los ciudadanos de las vulneraciones de los Derechos Humanos y, consecuentemente, el deber de remediar las dichas vulneraciones" (Carneiro Freira et al., 2013, p. 35). En este sentido, los Principios Rectores categorizan los mecanismos establecidos en estatales y no estatales, judiciales y no judiciales. Esto porque se evidencia que en la actualidad los mecanismos judiciales existentes no son suficientes; por ello, se proponen herramientas complementarias como: facilitación de información, arbitraje, negociación, investigación, mediación, conciliación, entre otras. No obstante, es responsabilidad del Estado construir un sistema integral de reparaciones. Carneiro Freire et al. (2013, p. 38) explican que esta

integralidad exige el pleno restablecimiento de las obligaciones de respeto y garantía mediante medidas de reparación que tiendan, no solo a borrar las huellas del impacto negativo en los derechos, sino también a evitar (y por tanto prevenir) la repetición de violaciones de los Derechos Humanos en el futuro, en especial cuando estas sean flagrantes.

Uno de los principales objetivos de los Principios Rectores es establecer un marco y directrices para construir una cultura y prácticas de prevención, mitigación, no repetición y reparación de impactos en los Derechos Humanos producidos por empresas (Organización de las Naciones Unidas, 2012).

Para revisar el avance de la implementación, el Alto Comisionado de las Naciones Unidas para los Derechos Humanos (ACNUDH) y el Grupo de Trabajo de las Naciones Unidas sobre Empresas y Derechos Humanos han convocado a dos consultas regionales para América Latina y el Caribe, donde reúnen a representantes del Estado, de la sociedad civil y de las empresas para revisar los logros y obstáculos en la implementación. En la segunda consulta realizada en enero de 2017, evidenciaron la urgencia de avanzar en políticas a nivel estatal, diferenciar el enfoque de empresas y Derechos Humanos del concepto de responsabilidad social empresarial, y de convocar a estos diálogos las comunidades que se han visto afectadas por operaciones empresariales. ${ }^{10}$ 


\section{MARCO ÉTICO - CARTA DE LA TIERRA}

Después de un diálogo intercultural de una década a nivel mundial en búsqueda de valores compartidos, en el año 2000, surge "La Carta de la Tierra", una declaración de principios éticos fundamentales para la construcción de una sociedad global justa, sostenible y pacífica. "La Carta reconoce que la protección ecológica, la erradicación de la pobreza, el desarrollo económico equitativo, el respecto a los Derechos Humanos, la democracia y la paz son interdependientes e indivisibles" (Carta..., 2000).

La metodología de construcción inclusiva y participativa de la Carta de la Tierra hace que tenga alta legitimidad y sea considerada con un estatus de ley blanda. Además, por su visión de esperanza y llamado a la acción, es un elemento de educación para el desarrollo sostenible. Adicionalmente, la UNESCO reconoce a través de la Referencia 32C/17 que es un importante marco ético para educar en el desarrollo sostenible. El preámbulo de La Carta inicia: "Estamos en un momento crítico de la historia de la Tierra, en el cual la humanidad debe elegir su futuro [...] Debemos unirnos para crear una sociedad global sostenible fundada en el respeto hacia la naturaleza, los derechos humanos universales, la justicia económica y una cultura de paz. En torno a este fin, es imperativo que nosotros, los pueblos de la Tierra, declaremos nuestra responsabilidad unos hacia otros, hacia la gran comunidad de la vida, y hacia las generaciones futuras" (Carta..., 2000). El Esquena 4 apunta los componentes de la Tierra.

La Carta tiene 4 pilares y 16 principios:

Figura 4: Componentes de La Carta de la Tierra

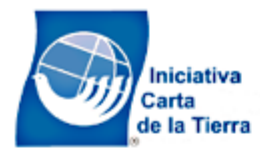

Valores y Principios para un Futuro Sostenible

RESPETO Y CUIDADO DE LA COMUNIDAD DE LA VIDA
1. Respetar la Tierra y la vida en toda su diversidad
2. Cuidar la comunidad de la vida con entendimiento, compasión y amor.
3. Construir sociedades democráticas que sean justas, participativas, sostenibles y pacificas
4. Asegurar que los frutos y la belleza de la Tierra se preserven para las generaciones presentes y futuras.
INTEGRIDAD ECOLOGICA
5. Proteger y restaurar la integridad de los sistemas ecológicos de la Tierra, con especial preocupación por la diversidad biológica y los procesos
naturales que sustentan la vida.
6. Evitar dañar como el mejor método de protección ambiental y cuando el conocimiento sea limitado, proceder con precaución.
7. Adoptar patrones de producción, consumo y reproducción que salvaguarden las capacidades regenerativas de la Tierra, los derechos humanos
y el bienestar comunitario.
8. Impulsar el estudio de la sostenibilidad ecológica y promover el intercambio abierto y la extensa aplicación del conocimiento adquirido.
JUSTICIA sOCIAL Y ECONÓMICA
9. Erradicar la pobreza como un imperativo ético, social y ambiental
10. Asegurar que las actividades e instituciones económicas, a todo nivel, promuevan el desarrollo humano de forma equitativa y sostenible.
11. Afirmar la igualdad y equidad de género como prerrequisitos para el desarrollo sostenible y asegurar el acceso universal a la educación, el
cuidado de la salud y la oportunidad económica.
12. Defender el derecho de todos, sin discriminación, a un entorno natural y social que apoye la dignidad humana, la salud fisica y el bienestar
espiritual, con especial atención a los derechos de los pueblos indigenas y las minorias.
DEMOCRACIA, NO VIOLENCIA Y PAZ
13. Fortalecer las instituciones democráticas en todos los niveles y brindar transparencia y rendimiento de cuentas en la gobernabilidad,
participación inclusiva en la toma de decisiones y acceso a la justicia
14. Integrar en la educación formal y en el aprendizaje a lo largo de la vida, las habilidades, el conocimiento y los valores necesarios para un
modo de vida sostenible.
15. Tratar a todos los seres vivientes con respeto y consideración
16. Promover una cultura de tolerancia, no violencia y paz.

Fuente: La carta... (2001).

La Carta de la Tierra hace un llamado urgente a las personas, organizaciones, empresas, gobiernos e instituciones transnacionales para tener una visión compartida sobre valores básicos que permitan alcanzar una vida sostenible. De manera particular, las empresas pueden avalar La Carta y con ello declaran que: 


\section{ANO 15 • NÚMERO 28 • 1ํㅗㅌ. 2018 • ORGANICOM}

\section{INVOLUCRAMIENTO DE LAS ORGANIZACIONES EN LA CONSTRUCCIÓN DE PAZ}

Adoptamos el espíritu y los objetivos del documento. Nos comprometemos a unirnos a la alianza mundial para un mundo justo, sostenible y pacífico, y a trabajar para la realización de los valores y de los principios de la Carta de la Tierra (declaración de aval). (Carta..., 2000)

\section{INICIATIVA -GLOBAL COMPACT-BUSSINESS FOR PEACE}

El Pacto Global, de acuerdo con la Figura 5, es una iniciativa de Naciones Unidas que se define como un movimiento global de empresas sostenibles, busca alinear las estrategias y operaciones de las empresas con diez (10) principios que se dividen en cuatro (4) áreas derivadas de Declaraciones y Convenciones, "Derechos Humanos, Normas laborales, Medio Ambiente y Anti-corrupción:

Figura 5: Principios del Global Compact

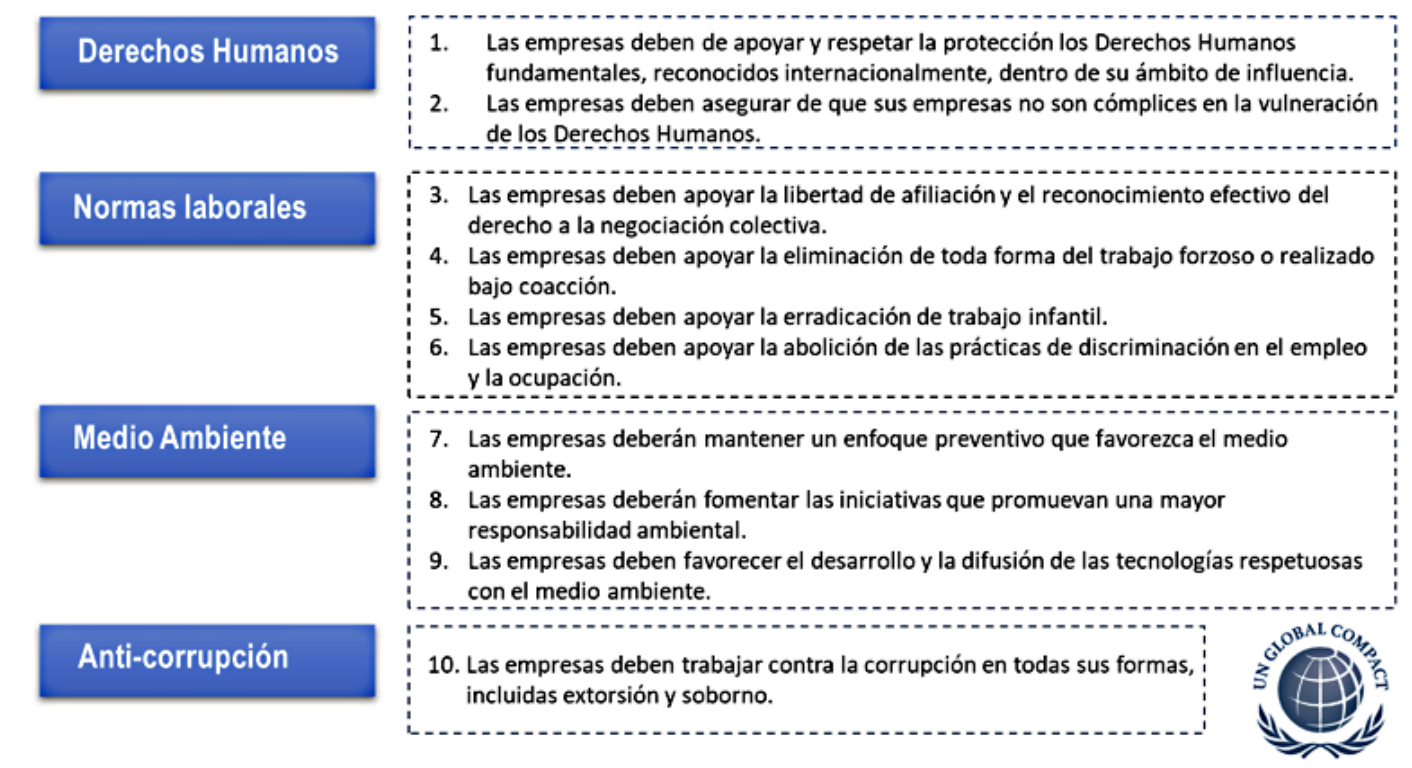

Fuente: recuperado de https://www.unglobalcompact.org.

El Global Compact cuenta con varias líneas de trabajo, aquí se mencionan dos de ellas, direccionadas a aportar herramientas para el cumplimiento de los Objetivos de Desarrollo Sostenible y, de manera específica, a la construcción de la paz. Estas son: Liderazgo empresarial - Blueprint y Empresas por la paz (Business for Peace).

\section{Blueprint - Liderazgo empresarial para los ODS (Blueprint for Business Leadership on the Sdgs)}

El primero de ellos, y presentado en la Cumbre de Líderes del Pacto Mundial de la ONU 2017, es una guía para fortalecer el liderazgo de todo tipo de organizaciones, con el fin de que estas aporten de manera global y sistémica para impactar a gran escala al logro de los ODS. Propone que un buen liderazgo debe llevar a la acción, por ello plantea que las empresas deben implementar un ciclo de gestión de tres pasos: PRIORIZAR, lo cual implica que esta evalúe sus mayores riesgos y aplique prácticas que la lleven al segundo paso que es ACTUAR para aportar de manera efectiva a los ODS, y, el tercer paso, APRENDER al conocer el impacto de sus acciones para luego documentar las lecciones aprendidas y compartirlas con el objeto de construir conocimiento colectivo. El Blueprint propone 5 características de un liderazgo empresarial: INTENCIONALIDAD, para que la estrategia de la empresa esté alineada deliberadamente con los ODS; AMBICIOSO, para superar las expectativas y tener visión de largo plazo; CONSISTENTE, para que se incorpore de forma sistémica al quehacer y ser de la empresa; COLABORATIVO, 
al integrar a todos sus grupos de interés; y RESPONSABLE, al ser transparente, comprometida y proactiva frente a los sus impactos positivos y negativos que genere su operación (Global Report Initiative; Pacto Global de las Naciones Unidas; World Business Council for sustainable Development, 2017).

\section{Empresas por la paz (Business for Peace)}

En el 2013, surge "Empresas por la Paz",12 para promover la paz en el lugar de trabajo, en el mercado, en las comunidades locales donde opera la organización, y, de manera especial, clarificar el rol que las empresas deben desempeñar en entornos de conflictos o de alto riesgo (conflictos violentos, riesgos sociales y políticos, abuso de derechos humano).

En el informe del United Nations Global Compact (2015) Avanzando en el desarrollo sostenible objetivos al respaldar la paz, se define que el rol de las empresas en entornos de conflictos es "evitar efectos negativos y contribuir al cambio positivo donde sea posible para abordar los principales impulsores del conflicto y de la violencia". Así mismo, propone unos niveles de compromiso e involucramiento empresarial frente a estos contextos; se explica en la Figura 6.

Figura 6: Espectro del compromiso empresarial frente a contextos afectados por conflictos - resumen del documento Avanzando en el desarrollo sostenible Objetivos al respaldar la paz, de Global Compact

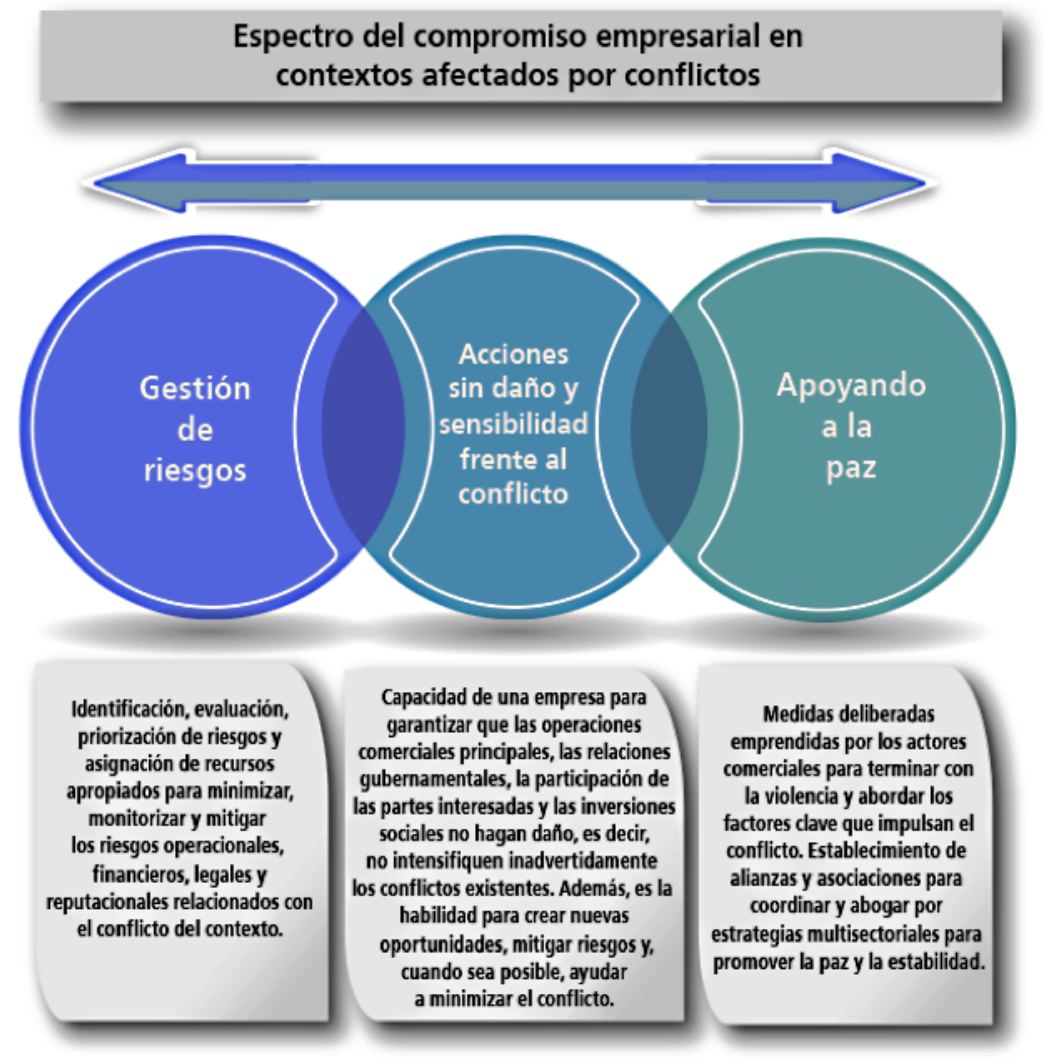

Fuente: adaptado de United Nations Global Compact (2015).

En este mismo sentido, el Global Compact ha desarrollado lineamientos sobre responsabilidad empresarial en áreas de alto riesgo y afectadas por conflictos para ayudar a las empresas a implementar prácticas empresariales responsables en estas áreas, y su enfoque es:

La responsabilidad de paz, seguridad y desarrollo es principalmente del gobierno, sin embargo, el sector privado puede hacer una contribución relevante a la estabilidad y seguridad en áreas afectadas por conflictos y de alto riesgo. Las actividades comerciales

12 Descripción tomada del United Nations Global Compact (2017) y <https:/www.pactoglobal-colombia.org/b4p/business-for-peace.html>. 


\section{ANO 15 • NÚMERO 28 • 1ํㅗㅌ. 2018 • ORGANICOM}

\section{INVOLUCRAMIENTO DE LAS ORGANIZACIONES EN LA CONSTRUCCIÓN DE PAZ}

tienen impactos positivos directos e indirectos al crear oportunidades de empleo, generar ingresos que hagan avanzar el desarrollo y la recuperación de las economías, hacer inversiones sustentables en ciudades y pueblos, crear políticas de contratación incluyentes que formen buenas relaciones entre las etnias y las comunidades, desarrollar estrategias de negocios de "base de la pirámide", y promover mejores prácticas en las áreas de derechos humanos, trabajo, ambiente y anticorrupción. Los negocios pueden también ser un incentivo poderoso para reunir a las personas de diversas nacionalidades y culturas, que generen relaciones basadas en un sentido compartido de identidad y propósito, superando las diferencias que, en la sociedad más amplia, son más difíciles de superar. (Oficina del Pacto Mundial de las Naciones Unidas, 2010, p.6)

A "Empresas para la paz" pertenecen 150 empresas líderes y asociaciones empresariales de 37 países ${ }^{13}$ que buscan identificar riesgos, participar en el diálogo público-privado, alinear estrategias de negocios a buenas prácticas, compartir innovación, y demostrar liderazgo en los avances con resultados concretos. Todo esto para convertir a las empresas en defensoras de la paz, entre y con todos sus grupos de interés (pares, colaboradores, clientes, proveedores, inversores, comunidad, gobierno, entre otros).

Adicionalmente, El Global Compact a partir del 2016, desde la iniciativa de "Empresas por la paz", impulsa una nueva perspectiva sobre las contribuciones deliberadas a la paz por parte de las empresas, aportando directamente al Objetivo de Desarrollo Sostenible 16, que busca promover sociedades, justas, pacíficas e inclusivas. El enfoque de esta perspectiva se fundamenta en que

para lograr la paz, la justicia y la inclusión, es importante que los gobiernos, la sociedad civil y las comunidades trabajen juntos para poner en práctica soluciones duraderas que reduzcan la violencia, hagan justicia, combatan eficazmente la corrupción, y garanticen en todo momento la participación inclusiva. (Organización de las Naciones Unidas, 2015, 0DS16)

\section{CONSIDERACIONES FINALES EL PAPEL DE LA COMUNICACIÓN ORGANIZACIONAL EN LA CONSTRUCCIÓN DE PAZ}

La comunicación es el eje transversal para recomponer el tejido social y recuperar la confianza para consolidar procesos de construcción de paz. Para ello, la visión de la gestión comunicacional debe evolucionar de la labor informativa a la relacional, en este sentido se proponen los siguientes roles a tener presentes.

\section{Interrelación sociedad-organización}

La comunicación es interacción y permite a las organizaciones comprender su entorno en el cual operan, y el nivel de comprensión de este contexto determina en gran medida la posibilidad de involucramiento de las empresas en la construcción de la paz. La falta de comprensión de la interrelación sociedad-organización es la que ha llevado a que los esfuerzos realizados por muchas empresas en mejorar las consecuencias sociales y medioambientales de sus actividades no sean productivos. Sea cual sea la iniciativa de realizar acciones de responsabilidad; por obligación moral o por la búsqueda de la sostenibilidad, o el otorgamiento de la licencia para operar, o el incremento de reputación corporativa, ya no son suficientes frente a los crecientes riesgos y necesidades que demanda la sociedad actual. (Porter; Kramer, 2006).

En este sentido la gestión comunicacional debe ir más allá de estructurar un mapa de públicos a realizar un monitoreo y caracterización permanente del contexto al implementar la metodología de análisis PASTE -aspectos políticos, socioculturales, tecnológicos y económicos (Fuentes, 2007, p. 83).

13 Dato obtenido del United Nations Global Compact (2017). 


\section{Construcción de vínculos basados en confianza}

Se ha mencionado que un factor crítico de éxito es la construcción de confianza porque es la base para crear relaciones. Para Lederach (2016, p. 85), "la construcción de paz exige la conciencia de la relación como la capacidad de imaginarse con el otro, de reconocerse, y que ello puede quebrar la violencia". Esta mirada relacional abre la posibilidad de presentar el gran aporte que tiene la comunicación organizacional en este escenario de construcción de la paz. Las empresas aún no han descubierto todo el potencial que tiene la comunicación, porque la han limitado a la gestión mediática e informativa; más aún, están por descubrir la capacidad que tiene para construir relaciones, consolidar la confianza y fortalecer la convivencia pacífica teniendo como base los Derechos Humanos.

El reto, entonces, es crear vínculos, lo cual "significa generar relaciones sólidas, fluidas y basadas en objetivos específicos que puedan perdurar en el tiempo y que le permitan a la organización generar un espacio de intercambio con sus diferentes públicos" (Manucci, 2004, p. 66).

\section{Una nueva narrativa de la paz}

En un proceso de transición hacia la construcción de paz, lo primero que se debe desarmar son las palabras y convertir el lenguaje en el vehículo de movilización donde se proporcionen argumentos que, a la vez, direccione las acciones hacia la paz: reconstruir relaciones, retomar más que recordar, reconocer para redescubrir, reconciliar para reencontrar y recuperar el respeto:

- Reconstruir relaciones es entrar en la dimensión del OTRO, según Manuel Ramiro Muñoz, director del Centro de Estudios Interculturales de la Pontificia Universidad Javeriana de Cali, lo define como "construirnos más allá de nuestra individualidad, pensando en un colectivo, en búsqueda de un bien común, justicia y solidaridad".

- Retomar más que recordar. Construir la paz implica mirar hacia el futuro, más que hacia el pasado; así expone Frederik de Klerk, Premio Nobel de la Paz en 1993, al referirse a su experiencia en Sudáfrica: "Nuestra incapacidad para llegar a un acuerdo sobre el pasado ha sido una de las fallas más grandes de nuestra sociedad del posconflicto. El pasado sigue interviniendo como una barrera invisible y, en casi todos los discursos nacionales, da el combustible para continuar la recriminación, la culpa y la polarización".

- Reconocer para redescubrir. Reconocer es incluir, para el Dr. Ramiro Muñoz, en el siglo XX se instauró una nueva forma de exclusión: la invisibilidad y la negación. Esto ha llevado a que algunos grupos queden rezagados y desconocidos de varios ámbitos de la sociedad. El reto es redescubrir las diferencias en un marco de respeto a la diversidad para construir desde la interculturalidad.

- Reconciliar para reencontrarse es construir puentes para pasar de los desencuentros a encuentros a partir de un diálogo basado en la escucha para llegar a acuerdos de convivencia pacífica y, por ende, a la unión. Pero este diálogo debe trascender a la espiritualidad, como decía San Juan Pablo II: "la verdadera reconciliación entre hombres enfrentados y enemistados solo es posible si se dejan reconciliar al mismo tiempo con Dios".

- Recuperar el respeto como la base de la paz, no solo con los seres humanos, sino también con la naturaleza, con nuestra casa común. La industrialización, el consumismo y la falta de conciencia en el cuidado y protección del medio ambiente han puesto en peligro la vida misma. Maya Lin, artista y arquitecta estadounidense, dice: "Creo que la paz solo llegará cuando aprendamos a vivir en este planeta de modo que permitamos a otras criaturas existir junto con nosotros" (Fuentes, 2016). 
Además de estos argumentos-acción, la relevancia de la comunicación está en pasar del Yo al Nosotros como una evolución corporativa. Evolucionar de la primera persona del singular, YO, a la primera persona del plural NOSOTROS, esto implica un cambio de conciencia como seres humanos y como sociedad. Como decía la Madre Teresa de Calcuta: "Si hoy no tenemos paz, es porque hemos olvidado que nos pertenecemos unos a otros, que aquel hombre, aquella mujer y aquel niño, son mi hermano y mi hermana".

La comunicación permite movilizar para pasar del dicho al hecho, posibilita recobrar el sentido de vivir en paz y de construir en conjunto un futuro sostenible donde se entre en otra sintonía para estar en la misma frecuencia, que permita armonizar y movilizar la paz anhelada y soñada.

\section{REFERENCIAS}

BLOOMFIELD, David; BARNES, Teresa; HUYSE, Luc (Eds.). Reconciliation after violent conflict. a handbook. Stockholm: International Institute for Democracy and Electoral Assistance, 2003. (Handbook Series).

BRUNDTLAND, Gro. Nuestro futuro común: comisión mundial sobre el medio ambiente y el desarrollo. Nueva York: ONU, 1987. Disponible en: <http://bit.ly/2NCZ5Tk>. Acceso en: 24 jul. 2018.

CARNEIRO FREIRE, Mar et al. Cuaderno guía de los principios rectores ONU sobre empresas y derechos humanos. Madrid: Sustentia Innovación Social, 2013.

CARTA de la Tierra. San José: Secretaria Internacional de la Carta de la tierra, 2000. Disponible en: <http://bit.ly/2LgBkE1>. Acceso en: 24 jul. 2018.

COMISIÓN HISTÓRICA DEL CONFLICTO Y SUS VÍCTIMAS. Contribución al entendimiento del conflicto armado en Colombia. Equipo Paz Gobierno, Bogotá, 10 feb. 2015. Disponible en: <http://bit.ly/2v3ki15>. Acceso en: 24 jul. 2018.

CONEXIÓN COP. Ban Ki-moon urge a las empresas colaborar en la lucha contra cambio climático. Conexión COP, [S.l.], 13 abr. 2015. Disponible en: <http://bit.ly/2MchhqA>. Acceso en: 13 ago. 2018.

CORPORACIÓN LATINOBARÓMETRO. La confianza en América Latina 1995-2015, 20 años de opinión pública. Latinobarómetro, Providencia, 2016. Disponible en: < http://bit.ly/2vNDr8H>. Acceso en: 4 ago. 2018.

Informe 2017. Latinobarómetro, Providencia, 2017. Disponible en: <http://bit.ly/2w6dNLB>. Acceso en: 4 ago. 2018.

DE ROUX, Stéphane J. F. Vamos a remendar este país: comunicación para la reconciliación - Lección inaugural de la Facultad de Comunicación y Lenguaje de la Pontificia Universidad Javeriana, Escola de Cultura de Pau. En: ESCOLA DE CULTURA DE PAU. Anuario de procesos de paz Vicenc Fisas, 2016. Barcelona: Icaria, 2016. Disponible en: <http://bit.ly/2LKyx26>. Acceso en: 14 jul. 2018.

FISAS, Vicenc. La paz es posible. Barcelona: Plaza \& Janés, 2002.

FUENTES, Sandra. Sistema de gestión comunicacional para la construcción de una marca ciudad o marca país. Revista Signo y Pensamiento, Bogotá, n. 51, p. 81-97, 2007. 
. Argumentos de paz. La República, Bogotá, 10 mar. 2016. Disponible en: <http://bit.ly/2LlBr11>. Acceso en: 24 jul. 2018.

GALTUNG, Johan. Sobre la paz. Barcelona: Fontamara, 1985.

GLOBAL REPORTING INITIATIVE; PACTO GLOBAL DE LAS NACIONES UNIDAS; WORLD BUSINESS COUNCIL FOR SUSTAINABLE DEVELOPMENT. SDG Compass. la guía para la acción empresarial en los ODS. [S.L.]: GRI; Pacto Global; WBCSD, 2015. Disponible en: <http://bit.ly/2LGOKFl>. Acceso en: 24 jul. 2018.

Blueprint for business leadership on the SDGS. [S.L.]: GRI; Pacto Global; WBCSD, 2017. Disponible en: <http://bit.ly/2NHzSr2>. Acceso en: 24 jul. 2018.

LA CARTA de la Tierra. Earth Charter Inaction, [S.L.], 1 feb. 2001. Disponible en: <http://bit.ly/2LImLo0>. Acceso en: 24 jul. 2018.

LEDERACH, John. Construyendo la paz. reconciliación sostenible en sociedades divididas. Bilbao: Gernika Gogoratuz, 1997.

La imaginación moral: el arte y el alma de la construcción de la paz. Bogotá: Libros Semana, 2016.

MANUCCI, Marcelo. Comunicación corporativa estratégica. Bogotá: Grupo Saf, 2004.

NILSON, Marcus; KREUTZ, Joakim. Protracted conflicts: issues or dynamics at stake? New Rules, [S.l.], v. 15, n. 4, p. 3-6, 2010.

OFICINA DEL PACTO MUNDIAL DE LAS NACIONES UNIDAS. Lineamientos sobre responsabilidad empresarial en áreas de alto riesgo y afectadas por conflictos. recursos para empresas e inversionistas. Nueva York: Global Compact, 2010. Disponible en: <http://bit.ly/2vlUYsq>. Acceso en: 24 jul. 2018.

ORGANIZACIÓN DE LAS NACIONES UNIDAS. Oficina del Alto Comisionado de Derechos Humanos. Principios rectores sobre las empresas y los derechos humanos. Nueva York: ONU, 2011. Disponible en: <http://bit.ly/2MckwhQ>. Acceso en: 4 ago. 2018.

El futuro que queremos. In: CONFERENCIA DE LAS NACIONES UNIDAS SOBRE EL DESARROLLO SOSTENIBLE, 2012, Rio de Janeiro. Documento final de la conferencia. Nueva York: ONU, 2012. Disponible en: <http://bit.ly/2A4TQKa>. Acceso en: 24 jul. 2018.

Transformar nuestro mundo: la agenda 2030 para el desarrollo sostenible. Nueva York: ONU, 2015. Disponible en: <http://bit.ly/2JQl1rO>. Acceso en: 24 jul. 2018.

Objetivos de desarrollo sostenible. Naciones Unidas, Nueva York, [s.d.]. Disponible en: <http://bit.ly/2LLX5b5>. Acceso en: 24 jul. 2018.

PORTER, Michael; KRAMER, Mark. Estrategia y sociedad. Harvard Business Review América Latina, Boston, reimp. R0612D-E, p. 3-15, dic. 2006.

RETTBERG, Angelika. La empresa privada en la construcción de la paz en Colombia. In: FORO COLOMBIANO EN CONSTRUCCIÓN DE PAZ, 1., 2011, Bogotá. Actas... Bogotá: UTadeo, 2011. Disponible en: <http://bit.ly/2uYtsMv>. Acceso en: 24 jul. 2018. 
RETTBERG, Angelika.Empresa y paz. La República, Bogotá, 11 de septiembre 2012. Disponible en: https://www.elespectador. com/opinion/empresa-y-paz. Acceso en: 24 jul. 2018.

ROJAS, Francisco. Confianza, ¿un bien escaso? Buenos Aires: Teseo, 2011.

SAVATER, Fernando. Ética para las empresas. Barcelona: Peguin Random House, 2014.

UNITED NATIONS GLOBAL COMPACT. Advancing the sustainable development goals by supporting peace: how business can contribute. United Nations Global Compact, Nueva York, 2015. Disponible en: <http://bit.ly/2A4u2hj>. Acceso en: 24 jul. 2018.

Business advancing peace. United Nations Global Compact, Neuva York, 2017. Disponible en: <http://bit.ly/2LLERWZ>. Acceso en: 14 jul. 2018.

Artículo recibido el 10.05.2018 y aprobado el 20.06.2018. 